\title{
A Generalization of Multiple-Mode OFDM with Index Modulation
}

\author{
Miaowen $\mathrm{Wen}^{\dagger}$, Qiang $\mathrm{Li}^{\dagger}$, Ertugrul Basar ${ }^{\ddagger}$, and Wensong Zhang ${ }^{\dagger}$ \\ ${ }^{\dagger}$ School of Electronic and Information Engineering, \\ South China University of Technology, Guangzhou 510640, China \\ ${ }^{\ddagger}$ Communications Research and Innovation Laboratory (CORELAB), \\ Department of Electrical and Electronics Engineering, Koç University, Sariyer 34450, Istanbul, Turkey \\ Email: eemwwen@scut.edu.cn, \{eeqiangli, eez.wensong\}@ mail.scut.edu.cn, ebasar@ku.edu.tr
}

\begin{abstract}
In this paper, we propose the scheme of generalized multiple-mode orthogonal frequency division multiplexing with index modulation (GMM-OFDM-IM), which allows a different subcarrier to utilize a signal constellation of a different size while conveying the same number of IM bits. Considering phase shift keying (PSK) constellations, we present design guidelines for GMM-OFDM-IM to achieve the optimal error performance in the asymptotically high signal-to-noise ratio region. A computationally efficient and near-optimal detector based on the idea of sequential decoding is also tailored to GMM-OFDM-IM to avoid the detection of an illegimate constellation permutation. Monte Carlo simulations are conducted to examine GMM-OFDM-IM, whose inherent properties and advantages are revealed by the simulation results.

\section{INTRODUCTION}

IM is a novel digital modulation concept that relies on the indices of some building blocks of communication systems to convey information [1]. For its appealing advantages such as superior bit error rate (BER) performance, IM has been widely recognized as a candidate modulation technique for nextgeneration wireless networks [2], [3]. Recently, a great deal of effort has been poured into developing a special realization of IM by exploring the OFDM subcarriers. In OFDM-IM [4], the total OFDM subcarriers are divided equally into several subcarrier groups to perform IM independently, where the size of each group is denoted by $n$. For each subcarrier group, the information bits are loaded onto both the conventional $M$-ary modulation symbols carried on $a$ active subcarriers and the subcarrier activation patterns of number $2^{\left\lfloor\log _{2} C(n, a)\right\rfloor}$, where $C(\cdot, \cdot)$ and $\lfloor\cdot\rfloor$ stand for the binomial coefficient and the floor function, respectively.

More recently, great attention has been paid to the use of multiple signal constellations for IM. The first example with two signal constellations (primary and secondary ones) was presented in [5], where the entire signal processing procedure follows the one of OFDM-IM except that the "inactive" subcarriers transmit signals from a non-zero signal constellation (secondary one) that is distinguishable from the primary one utilized by the "active" subcarriers, rather than the null subcarriers as in OFDM-IM. The signal constellation was also referred to as a mode in [5], and thereby the proposed scheme therein was named dual-mode (DM-) OFDM. In [6], a novel MM-OFDM-IM scheme that modulates $n$ subcarriers by $n$ distinguishable signal constellations of the same size as well as their permutations was proposed and its diversity was further enhanced in [7]. Due to the capability of transmitting more IM bits with a permutational increase, MM-OFDM-IM is shown to achieve much higher spectral efficiency (SE) and better BER performance than both OFDM-IM and DM-OFDM [6]. It should be noted that MM-OFDM-IM is not a straightforward generalization of DM-OFDM. The direct extension of DMOFDM to multiple modes was in fact proposed in [8], where $n$ subcarriers are divided into $L$ subgroups $(L=2$ for DMOFDM) each transmitting a different mode.

Aiming to provide more flexible choices of SE values, in this paper, we propose a generalization of MM-OFDM-IM, termed as GMM-OFDM-IM, by considering PSK constellations and relaxing the constraint on the cardinalities of the signal constellations employed by different subcarriers. We present guidelines to obtain $n$ distinguishable signal constellations from a unit circle that lead to the optimal asymptotic BER performance of GMM-OFDM-IM systems. Also, we design a novel detector for GMM-OFDM-IM motivated by the sequential decoding idea. This detector avoids the decision on an illegal constellation permutation, thus achieving a performance closer to that of the maximum-likelihood (ML) detector with slightly increased computational complexity. ${ }^{1}$

\section{System Model of GMM-OFDM-IM}

The GMM-OFDM-IM transmitter, which is built on the conventional OFDM architecture of $N$ subcarriers, is depicted in Fig. 1. At the beginning, a total of $P$ incoming bits to be transmitted via an OFDM symbol are equally split into $g$ blocks, each containing $m=P / g$ bits, and correspondingly, the total OFDM subcarriers are divided into $g$ groups, each comprising $n=N / g$ subcarriers. Then, one bit block modulates one subcarrier group of a size much smaller than $N$ independently, reducing the implementation complexity of IM. For brevity, let us focus on the processing procedure associated with the $\gamma$-th bit block and subcarrier group, where $\gamma \in\{1, \ldots, g\}$. Specifically, the available $m$ bits are further

\footnotetext{
${ }^{1}$ Notation: Scalar variables are in italic letters. Column vectors and matrices are denoted by lowercase and uppercase boldface letters, respectively. Superscript ${ }^{T}$ stands for the transpose operation. $\|\cdot\|_{F}$ refers to the Frobenius norm. $\mathbf{I}_{n}$ denotes the $n \times n$ identity matrix. $\mathcal{C N}\left(\mu, \sigma^{2}\right)$ denotes the complex Gaussian distribution with mean $\mu$ and variance $\sigma^{2} \cdot \operatorname{diag}(\cdot)$ transforms a
} vector into a diagonal matrix. 


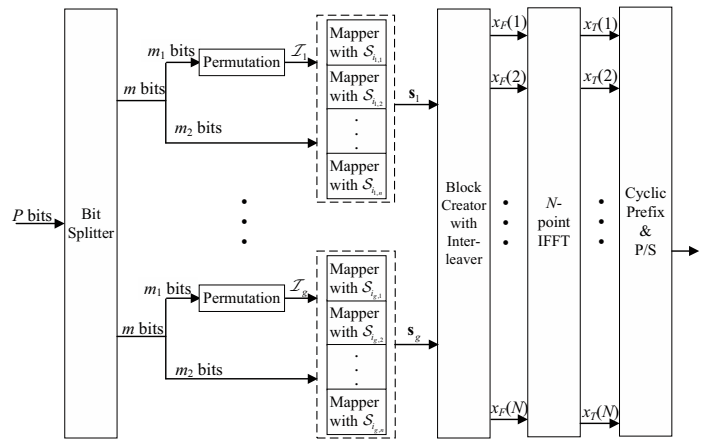

Fig. 1. Block diagram of GMM-OFDM-IM transmitter.

partitioned into two parts for different purposes. The first part, consisting of $m_{1}$ bits, determines the order of the sequence $\mathcal{Q}(=\{1, \ldots, n\})$, generating $\mathcal{I}_{\gamma}=\left\{i_{\gamma, 1}, \ldots, i_{\gamma, \beta}, \ldots, i_{\gamma, n}\right\}$, where $i_{\gamma, \beta} \in \mathcal{Q}$ with $\beta \in \mathcal{Q}$. Using $\mathcal{I}_{\gamma}$, the mode employed by the $\beta$-th subcarrier, denoted by $\mathcal{S}_{i_{\gamma, \beta}}$, is determined as the $i_{\gamma, \beta^{-}}$ th element of a mode set $\left\{\mathcal{S}_{1}, \ldots, \mathcal{S}_{n}\right\}$. It is noteworthy that to enable IM, we have to ensure that $\mathcal{S}_{\beta} \cap \mathcal{S}_{\beta^{\prime}}=\emptyset, \forall \beta, \beta^{\prime} \in$ $\mathcal{Q}, \beta \neq \beta^{\prime}$, though they may have different constellation sizes. Without loss of generality, we assume that $n_{k}$ out of $n$ modes are $M_{k}$-ary constellations, where $\sum_{k=1}^{K} n_{k}=n$ and $M_{1}>$ $M_{2}>\cdots>M_{K}$. In this paper, all modes are limited to be PSK constellations and the combination of the total $n$ modes is presented as an $\mathcal{M}$-ary constellation that is normalized to have unit average power, where $\mathcal{M}=\sum_{k=1}^{K} n_{k} M_{k}$. The second part, made up of $m_{2}=\sum_{k=1}^{K} n_{k} \log _{2} M_{k}$ bits, is mapped to an $n \times 1$ symbol vector $\mathbf{s}_{\gamma}=\left[s_{\gamma, 1}, \ldots, s_{\gamma, \beta}, \ldots, s_{\gamma, n}\right]^{T}$, where $s_{\gamma, \beta} \in \mathcal{S}_{i_{\gamma, \beta}}$. Since $\left\{\mathcal{S}_{i_{\gamma, 1}}, \ldots, \mathcal{S}_{i_{\gamma, n}}\right\}$ is a full permutation of $\left\{\mathcal{S}_{1}, \ldots, \mathcal{S}_{n}\right\}$ that has $n$ ! possible realizations, we have $m_{1}=\left\lfloor\log _{2} n !\right\rfloor$ and there are $n !-2^{\left\lfloor\log _{2} n !\right\rfloor}$ unused (illegal) permutations. The mapping between $m_{1}$ bits and $\mathcal{I}_{\gamma}$ can be easily implemented through a look-up table or the permutation method [6].

After $\mathbf{s}_{\gamma}$ for all $\gamma$ are obtained, they enter the block creator coupled with the subcarrier-level interleaver, outputting the $N \times 1$ main OFDM block

$$
\begin{aligned}
\mathbf{x}_{F} & =\left[x_{F}(1), \ldots, x_{F}(N)\right]^{T} \\
& =\left[s_{1,1}, \ldots, s_{g, 1}, s_{1,2}, \ldots, s_{g, 2}, \ldots, s_{1, n}, \ldots, s_{g, n}\right]^{T} .
\end{aligned}
$$

Then, $N$-point inverse fast Fourier transform (FFT) is performed on $\mathbf{x}_{F}$ to generate the time-domain signal $\mathbf{x}_{T}=$ $\left[x_{T}(1), \ldots, x_{T}(N)\right]^{T}$. A length- $G$ cyclic prefix $(\mathrm{CP})\left[x_{T}(N-\right.$ $\left.G+1), \ldots, x_{T}(N)\right]^{T}$ is further appended at the beginning of $\mathbf{x}_{T}$, and the resulting signal is fed into parallel-to-serial (P/S) and digital-to-analog converters successively. Finally, the output signal is emitted into the frequency-selective Rayleigh fading channel, whose time-domain channel impulse response is given by $\mathbf{h}_{T}=\left[h_{T}(1), \ldots, h_{T}(\nu)\right]^{T}$, where $\nu \leq G$ and each entry of $\mathbf{h}_{T}$ follows the distribution $\mathcal{C N}(0,1 / \nu)$. We denote the frequency-domain channel transfer function (CTF) by $\mathbf{h}_{F}=\left[h_{F}(1), \ldots, h_{F}(N)\right]^{T}$, which is the $N$ point FFT of $\overline{\mathbf{h}}_{T}$, where $\overline{\mathbf{h}}_{T}$ is a length- $N$ vector given by
$\overline{\mathbf{h}}_{T}=\left[\mathbf{h}_{T}^{T}, 0, \ldots, 0\right]^{T}$.

At the receiver, the $\mathrm{CP}$ is first removed from the received signal. Subsequently, $N$-point FFT and de-interleaving operations are carried out in sequence, yielding the frequencydomain received signal associated with the $\gamma$-th group

$$
\mathbf{y}_{\gamma}=\left[y_{\gamma}(1), \ldots, y_{\gamma}(n)\right]^{T}=\mathbf{X}_{\gamma} \mathbf{h}_{\gamma}+\mathbf{w}_{\gamma},
$$

where $\mathbf{X}_{\gamma}=\operatorname{diag}\left(\mathbf{s}_{\gamma}\right), \mathbf{h}_{\gamma}=\left[h_{F}(\gamma), h_{F}(\gamma+g), \ldots, h_{F}(\gamma+\right.$ $(n-1) g)]^{T}$ refers to the frequency response vector associated with the $\gamma$-th subcarrier group, and $\mathbf{w}_{\gamma}$ is the noise vector in the frequency domain with the distribution $\mathcal{C N}\left(\mathbf{0}, N_{0} \mathbf{I}_{n}\right)$. The average received SNR per subcarrier is defined as $\rho=$ $1 / N_{0}$. Note that after de-interleaving, the correlation among the elements of $\mathbf{h}_{\gamma}$ is significantly attenuated, and as a result it is reasonable to assume $\mathbf{h}_{\gamma} \sim \mathcal{C N}\left(\mathbf{0}, \mathbf{I}_{n}\right)$.

A straightforward solution to recover the transmitted bits is the ML detection, which can be formulated based on (2) as

$$
\left(\hat{\mathcal{I}}_{\gamma}, \hat{\mathbf{s}}_{\gamma}\right)=\underset{\mathcal{I}_{\gamma}, \mathbf{s}_{\gamma}}{\arg \min }\left\|\mathbf{y}_{\gamma}-\mathbf{X}_{\gamma} \mathbf{h}_{\gamma}\right\|_{F}^{2}
$$

where $\hat{\mathcal{I}}_{\gamma}$ and $\hat{\mathbf{s}}_{\gamma}$ are the estimates of $\mathcal{I}_{\gamma}$ and $\mathbf{s}_{\gamma}$, respectively. To decrease the computational burden to the receiver, we will derive a low-complexity detector in Section IV.

From above, without taking into account the CP cost, the SE of GMM-OFDM-IM systems, measured by bps/Hz, is given by

$$
\mathcal{F}=\frac{m_{1}+m_{2}}{n}=\frac{1}{n}\left(\left\lfloor\log _{2} n !\right\rfloor+\sum_{k=1}^{K} n_{k} \log _{2} M_{k}\right) .
$$

For given values of $n_{k}$ and $M_{k}, k=1, \ldots, K$, how to choose appropriate modes $\mathcal{S}_{1}, \ldots, \mathcal{S}_{n}$ that lead to the optimal BER performance and simple symbol modulation/demodulation are key issues for GMM-OFDM-IM systems. In the next section, we will solve this practical problem.

\section{Mode Selection fOR GMM-OFDM-IM}

In this section, we design guidelines for mode selection under the constraint that all subcarriers transmit with the same instantaneous power, which means all $\mathcal{M}$ signal points are located on a unit circle, i.e., $r_{1}=\cdots=r_{K}=1$, where $r_{k}$ is the radius of the $M_{k}$-ary circle constellation.

According to the analysis in [6], the optimal modes should maximize the minimum intra-mode distance (MIAD)

$$
\begin{array}{r}
d_{1}=\min _{\mu, v \in\left\{1, \ldots, 2^{m}\right\}}\left\|\mathbf{X}_{\gamma}^{(\mu)}-\mathbf{X}_{\gamma}^{(v)}\right\|_{F}^{2}, \\
\text { s.t. } \quad\left|s_{\gamma, \beta}\right|^{2}=1, \operatorname{rank}\left(\mathbf{X}_{\gamma}^{(\mu)}-\mathbf{X}_{\gamma}^{(v)}\right)=1,
\end{array}
$$

and the minimum inter-mode distance (MIRD)

$$
\begin{array}{r}
d_{2}=\min _{\mu, v \in\left\{1, \ldots, 2^{m}\right\}}\left\|\mathbf{X}_{\gamma}^{(\mu)}-\mathbf{X}_{\gamma}^{(v)}\right\|_{F}^{2}, \\
\text { s.t. } \quad\left|s_{\gamma, \beta}\right|^{2}=1, \operatorname{rank}\left(\mathbf{X}_{\gamma}^{(\mu)}-\mathbf{X}_{\gamma}^{(v)}\right)=2,
\end{array}
$$

where $\mathbf{X}_{\gamma}^{(\mu)}$ and $\mathbf{X}_{\gamma}^{(v)}$ are two different realizations of $\mathbf{X}_{\gamma}$. Recall that on a circle, the uniformly distributed signal points have the maximum minimum Euclidean distance (MED). 
Hence, from (5), the $M_{k}$-ary constellation should be the ordinary $M_{k}$-PSK constellation, which has an MIAD of $\sqrt{2-2 \cos \left(2 \pi / M_{k}\right)}$.

In (6), the second-order diversity is controlled by two error events: 1) any two symbols are erroneously detected as another two symbols belonging to the same two modes; 2) the permutation of any two modes is erroneously detected. The probability of the first error event depends on the MIAD and can be also minimized based on the criterion of (5). Therefore, with the result of (5) as the input, the problem of the MIRD maximization described in (6) is equivalent to the problem of the minimization of the probability of the second error event.

Since $M_{1} / M_{k}$ is always equal to an integer power of two, the $M_{1}$-PSK constellation subsumes any of the $M_{k^{-}}$ PSK constellations, where $k=2, \ldots, K$. Hence, in order to maximize the MED of the combined $\mathcal{M}$-ary constellation, the optimal $n$ modes must be taken from the regular $\eta M_{1}$ PSK constellation, which provides the maximum MIRD value of $\sqrt{2-2 \cos \left(2 \pi / \eta M_{1}\right)}$, where $\eta$ is the minimum integer satisfying $\eta M_{1} \geq \mathcal{M}$. For ease of implementation, it is preferred to derive $n_{k}$ different $M_{k}$-PSK constellations starting from $k=1$ to $k=K$ sequentially. The detailed procedure is summarized in Algorithm 1, where the mode of $M_{k}$ signal points is represented by $M_{k}$ indices of the signal points of the $\eta M_{1}$-PSK constellation. Note that two immediately adjacent signal points of a signal constellation are required to be given consecutive indices in Algorithm 1. For example, when

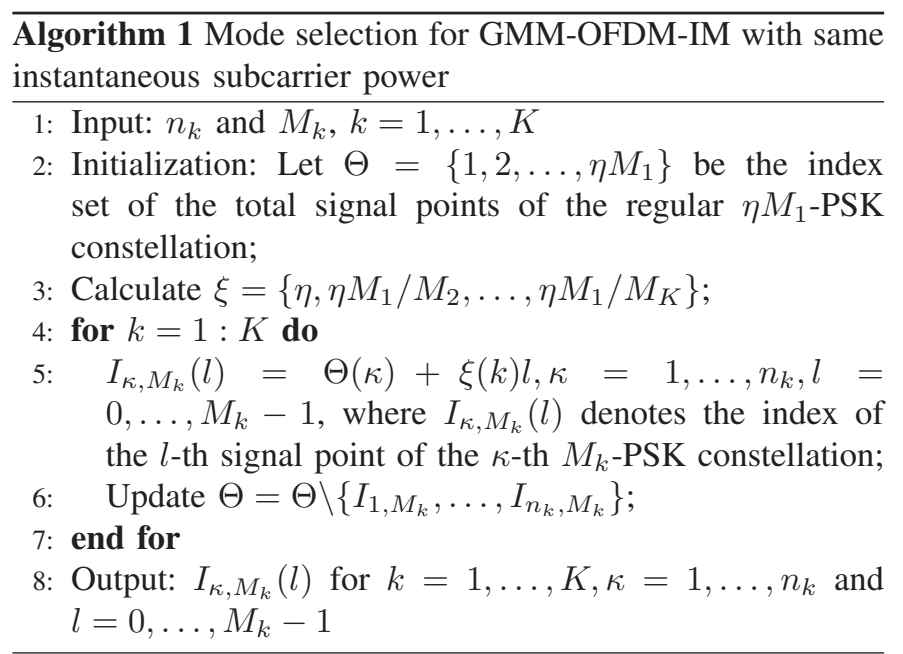

$\left(M_{1}\left(n_{1}\right), M_{2}\left(n_{2}\right), M_{3}\left(n_{3}\right)\right)=(8(1), 4(2), 2(1))$, it follows that $\eta=3$, and according to Algorithm 1, we can obtain the optimal four modes, which are depicted in Fig. 2(a).

\section{LOW-COMPlexity ReCEIVER DESIGN}

Motivated by the sequential decoding algorithm originally proposed for the convolutional codes [10], [11], in this section, we propose a low-complexity detector for GMM-OFDM-IM systems. Since the detection for each subcarrier group is identical and independent, in what follows, we will only focus on the $\gamma$-th subcarrier group and omit the subscript $(\gamma)$.

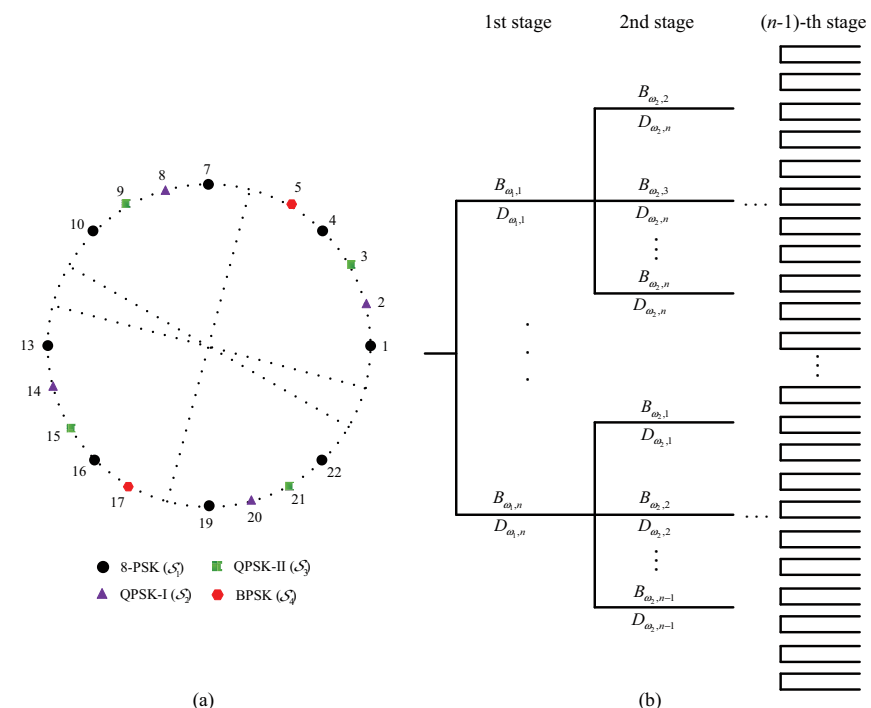

Fig. 2. (a) Four optimal modes with $\left(M_{1}\left(n_{1}\right), M_{2}\left(n_{2}\right), M_{3}\left(n_{3}\right)\right)=$ $(8(1), 4(2), 2(1))$; (b) Sequential detecting tree for GMM-OFDM-IM.

To begin with, we introduce an $n \times n$ matrix $\mathbf{B}$, whose $(\varsigma, \tau)$-th entry represents the most-likely transmitted symbol on the $\varsigma$-th subcarrier when it employs $\mathcal{S}_{\tau}$

$$
B_{\varsigma, \tau}=\underset{s \in \mathcal{S}_{\tau}}{\arg \min }|y(\varsigma)-s \cdot h(\varsigma)|^{2}, \quad \varsigma, \tau \in \mathcal{Q},
$$

and construct an $n \times n$ matrix $\mathbf{D}$ with

$$
D_{\varsigma, \tau}=\left|y(\varsigma)-B_{\varsigma, \tau} h(\varsigma)\right|^{2}
$$

to store the optimal metrics. Then, the CTFs of $n$ subcarriers are sorted according to their absolute values in decreasing order. Let us define $\Omega=\left\{\omega_{1}, \ldots, \omega_{n}\right\}$ for $\left|h\left(\omega_{1}\right)\right|^{2}>\cdots>$ $\left|h\left(\omega_{n}\right)\right|^{2}, \omega_{\beta}, \beta \in \mathcal{Q}$.

To apply the sequential detector, we build up the detecting tree in Fig. 2(b), which has $n-1$ stages following the order dictated in $\Omega$. In this tree, each node at the $(\beta-1)$-th stage has $n+1-\beta$ successors that represent $n+1-\beta$ possible modes carried on the $\omega_{\beta}$-th subcarrier along the path, where $\beta \in\{1, \ldots, n-1\}$, and each branch is labeled with the metric as well as the associated most-likely transmitted symbol. The aim of this sequential detector is to find a path from the tree that has the minimum cumulated metric and is legal. This search can be facilitated with a stack, of which each entry contains a path along with its cumulated metric and all entries are sorted in the order of increasing metric. The details of the proposed sequential algorithm can be outlined in the following five steps:

1) Calculate the matrices $\mathbf{B}$ and $\mathbf{D}$ by (7) and (8), respectively, and sort the CTFs of $n$ subcarriers to obtain $\Omega$;

2) Load the stack with the paths at the first stage of the tree and sort the stack in the order of increasing metric;

3) Replace the top path with its successors at the next stage of the tree, update the metric associated with the new paths, and rearrange the stack in the order of increasing metric; 


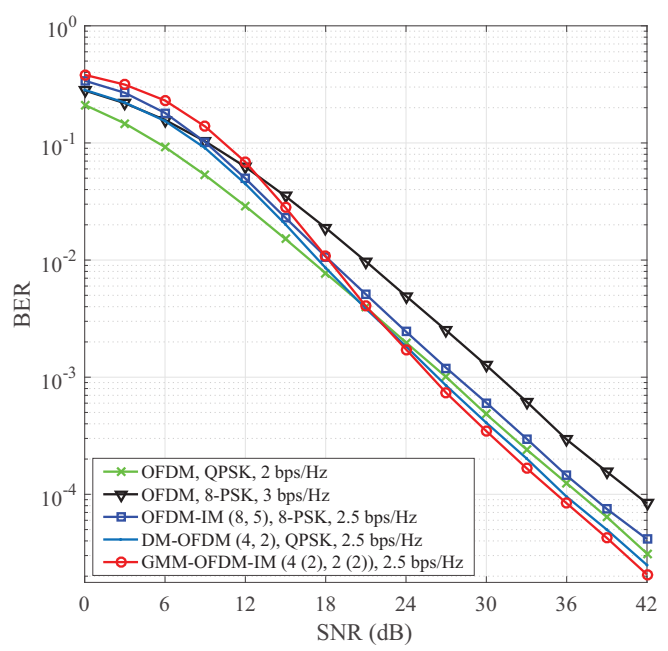

Fig. 3. Performance comparison among classical OFDM, OFDM-IM, DMOFDM, and GMM-OFDM-IM at an SE of $2.5 \mathrm{bps} / \mathrm{Hz}$.

4) If the length of the top path reaches $n-1$, supplement the remaining one mode; otherwise, return to Step 3);

5) Examine the legality of the top path by a look-up table or the permutation method. If it is legal, stop; otherwise, delete the top path and return to Step 3).

When the algorithm terminates, the top path in the stack is regarded as the estimated mode permutation. Meanwhile, the transmitted symbol on each subcarrier is obtained.

\section{Simulation Results and Discussion}

In this section, we perform Monte Carlo simulations to evaluate the BER performance of GMM-OFDM-IM systems and compare it with that of classical OFDM, OFDM-IM, and DM-OFDM systems. All considered schemes operate over Rayleigh fading channels and perfect channel estimation is assumed at the receivers. For notational simplicity, the OFDM-IM scheme with $a$ out of $n$ subcarriers being active and transmitting $M$-PSK symbols is referred to as "OFDM-IM $(n, a), M$-PSK", the DM-OFDM scheme with $a$ out of $n$ subcarriers employing the primary $M$-ary PSK constellation as "DM-OFDM $(n, a), M$-PSK", and the GMMOFDM-IM scheme with $n_{1}, \ldots, n_{K}$ subcarriers respectively employing $M_{1}, \ldots, M_{K}$-ary constellations as "GMM-OFDMIM $\left(M_{1}\left(n_{1}\right), \ldots, M_{K}\left(n_{K}\right)\right)$ ". The DM-OFDM scheme also adopts our design guidelines to generate the optimal primary and secondary constellations.

Fig. 3 presents the comparison results between classical OFDM, OFDM-IM, DM-OFDM, and GMM-OFDM-IM, where all involved schemes employ the optimal ML detection and achieve an SE of $2.5 \mathrm{bps} / \mathrm{Hz}$ except OFDM with QPSK (8PSK) that has an SE of 2 (3) bps/Hz. As can be observed from Fig. 3, all schemes achieve the same diversity order of unity. While achieving a higher SE, OFDM-IM performs slightly worse than classical OFDM with QPSK; however, it acquires an SNR gain of about $3 \mathrm{~dB}$ over classical OFDM with 8-PSK.

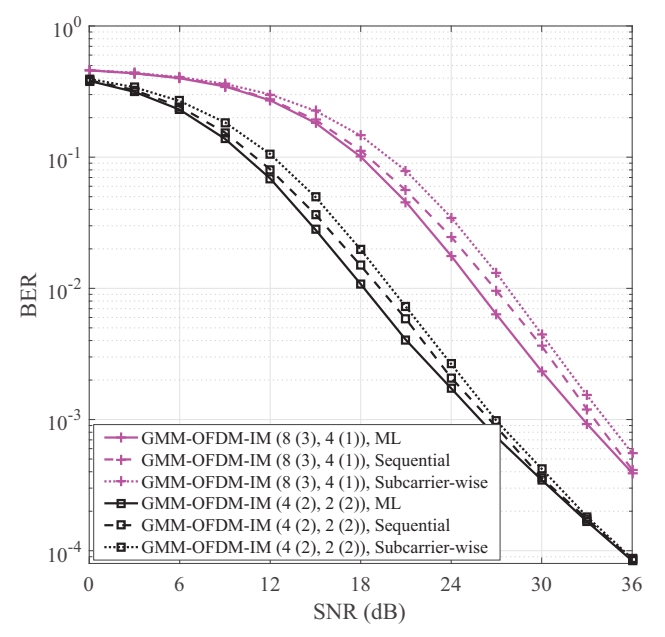

Fig. 4. Performance of the sequential detector for GMM-OFDM-IM.

Both DM-OFDM and GMM-OFDM-IM outperform classical OFDM and OFDM-IM in the medium-to-high SNR region, verifying the advantages of using multiple signal constellations for IM. However, thanks to the larger proportion of IM bits, GMM-OFDM-IM obtains approximately $1 \mathrm{~dB}$ SNR gain over DM-OFDM.

Fig. 4 illustrates the BER performance of the proposed sequential detectors, the subcarrier-wise detectors [6], and the optimal ML detectors for "GMM-OFDM-IM (8 (3), 4 (1))" and "GMM-OFDM-IM (4 (2), 2 (2))". It is observed from Fig. 4 that for all GMM-OFDM-IM systems, the sequential detectors perform better than the subcarrier-wise detectors for the entire SNR range. On the other hand, the sequential detector suffers from a performance loss of approximately $1.5 \mathrm{~dB}$ in comparison with the ML detector in the medium SNR region. However, at both low and high SNR the sequential detector has the capability of achieving near-optimal performance. Recalling that the computational complexity of the sequential detector is intermediate between that of ML and subcarrierwise detectors, the sequential detector provides a good tradeoff between the error performance and the complexity.

\section{CONCLUSION}

In this paper, a novel OFDM transmission scheme, namely GMM-OFDM-IM, which enjoys a more flexible SE than MMOFDM-IM by allowing a signal constellation of a different size to modulate different OFDM subcarriers, has been proposed. Design guidelines and a low-complexity near optimal detector for GMM-OFDM-IM have been given. Monte Carlo simulation results have demonstrated the superior performance of GMM-OFDM-IM over the existing frequency-domain IM techniques.

\section{ACKNOWLEDGEMENT}

This work was supported in part by the National Natural Science Foundation of China under Grant 61501190, in part by the Natural Science Foundation of Guangdong Province under Grant 2018B030306005, and in part by the Pearl River Nova Program of Guangzhou under Grant 201806010171. 


\section{REFERENCES}

[1] X. Cheng, M. Zhang, M. Wen, and L. Yang, "Index modulation for 5G: Striving to do more with less," IEEE Wireless Commun. Mag., Apr. 2018.

[2] E. Basar, M. Wen, R. Mesleh, M. Di Renzo, Y. Xiao, and H. Haas, "Index modulation techniques for next-generation wireless networks," IEEE Access, vol. 5, pp. 16693-16746, Sep. 2017.

[3] M. Wen, X. Cheng, and L. Yang, Index Modulation for $5 G$ Wireless Communications. Berlin: Germany, Springer, 2017.

[4] E. Basar, U. Aygolu, E. Panayirci, and H. V. Poor, "Orthogonal frequency division multiplexing with index modulation," IEEE Trans. Signal Process., vol. 61, no. 22, pp. 5536-5549, Nov. 2013.

[5] T. Mao, Z. Wang, Q. Wang, S. Chen, and L. Hanzo, "Dual-mode index modulation aided OFDM," IEEE Access, vol. 5, pp. 50-60, Feb. 2017.

[6] M. Wen, E. Basar, Q. Li, B. Zheng, and M. Zhang, "Multiple-mode orthogonal frequency division multiplexing with index modulation," IEEE Trans. Commun., vol. 65, no. 9, pp. 3892-3906, Sep. 2017.

[7] Q. Li, M. Wen, E. Basar, H. V. Poor, B. Zheng, and F. Chen, "Diversity enhancing multiple-mode OFDM with index modulation," IEEE Trans. Commun., to be published.

[8] Q. Li, M. Wen, H. V. Poor, and F. Chen, "Information guided precoding for OFDM,” IEEE Access, vol. 5, pp. 19644-19656, Oct. 2017.

[9] M. K. Simon and M.-S. Alouini, Digital Communication over Fading Channels, Second Edition. New York: Wiley, 2005.

[10] J. M. Wozencraft and B. Reiffen, Sequential Decoding. MIT Press, Cambridge, 1961.

[11] R. M. Fano, "A heuristic discussion of probabilitic decoding," IEEE Trans. Inform. Theory, vol. IT-9, pp. 64-74, Apr. 1963. 